\title{
Selection of Possible Code Words in Channel Coding Systems
}

\author{
Yong-Geol Shim \\ Department of Electronics Engineering, Dankook University, \\ 119, Dandae-ro, Dongnam-gu, Cheonan \\ Chungnam, 330-714, Korea \\ ygshim@dankook.ac.kr
}

\begin{abstract}
A selection method of possible code words in channel coding systems is presented. The proposed method is for channel coding of digital communication systems. Measuring the error correction capability with reunion junction, the proposed method has superior performance and low implementation complexity.
\end{abstract}

Keywords: Channel coding, Error correcting codes

\section{Introduction}

There are many variations in individual and typically proprietary implementations of synchronization and demodulation algorithms [1]. The focus is on developing principles, and on providing a selection method of possible code words in channel coding systems.

Good transceiver designs are often based on a sound understanding of such principles, together with a willingness to make approximations guided by intuition, driven by implementation constraints, and verified by simulations.

In this paper, a selection method of possible code words [2,3] in channel coding systems is presented. The proposed method is for channel coding of digital communication systems. Measuring the error correction capability with reunion junction, the proposed method has superior performance and low implementation complexity.

Given the equivalence of pass-band and complex baseband, it is understandable why, in typical modern pass-band transceivers [4], the intelligence is moved to baseband processing.

For moderate bandwidths at which analog-to-digital and digital-to-analog conversion can be accomplished inexpensively, baseband operations can be efficiently performed in DSP. These digital algorithms are independent of the pass-band over which communication eventually occurs, and are amenable to a variety of low-cost implementations, including very large scale integrated circuits (VLSI), field programmable gate arrays (FPGA), and general purpose DSP engines. On the other hand, analog components such as local oscillators, power amplifiers, and low noise amplifiers must be optimized for the bands of interest, and are often bulky. Thus, the trend in modern transceivers is to accomplish as much as possible using baseband DSP algorithms. For example, complicated filters shaping the transmitted waveform to a spectral mask dictated by the FCC can be achieved with baseband DSP algorithms, allowing the use of relatively sloppy analog filters at pass-band. Another example is the 
elimination of analog phase locked loops for carrier synchronization in many modern receivers.

The receiver instead employs a fixed analog local oscillator for down-conversion, followed by a digital phase locked loop implemented in complex baseband. Typically, the receiver would estimate the coefficients using the down-converter output.

\section{A Selection Method of Possible Code Words}

As with finite-energy signals, the inverse Fourier transform has the interpretation of autocorrelation. The power spectral density is given by

$$
S_{S}(f)=\frac{\left|S_{T_{0}}(f)\right|}{T_{0}}
$$

where the approximation neglects edge effects as the time interval gets large.

The correlation function is given by

$$
R_{S}(\tau)=\frac{1}{T_{0}} \int_{-\infty}^{\infty} S_{T_{0}}(z) S_{T_{0}}(z-\tau) d z
$$

An alternative method for computing PSD, therefore, is first to compute the empirical autocorrelation function. This is typically done in discrete time. While these methods are equivalent in theory, the properties of the estimates depend on a number of computational choices.

We therefore model the bit stream as random, which means that the modulated signal is modeled as a random process. Since the bit stream can be arbitrarily long, the energy of the modulated signal is unbounded.

When averaged over a long interval, the power of the modulated signal is finite, and tends to a constant, regardless of the transmitted bit stream. We must extend our discussion of baseband and pass-band signals to random processes. Random processes serve as a useful model not only for modulated signals, but also for noise and interference.

Let us now simplify the preceding expression and take the limit. Define the signal

$$
A(t)=\sum_{n} b[n] \mathrm{e}^{-j 2 \pi f n t} .
$$

Now, suppose that the symbols are uncorrelated, in that the time average is zero. Then the limit becomes

$$
\lim _{N \rightarrow \infty} \frac{A(t)}{N}=\sigma_{b}^{2} .
$$


Thus, we need the bits in a realization to exhibit enough variation. In practice, we often use line codes or scramblers specifically designed to avoid long runs of zeros or ones, in order to induce enough transitions for proper operation of synchronization circuits. The pass-band signal

$$
r_{p}(t)=\operatorname{Re}\left[r(t) e^{j 2 \pi f_{c} t}\right]
$$

goes through the channel. The pass-band rating signal is given by

$$
x_{p}(t)=A r_{p}(t-\tau)+n_{p}(t)
$$

where $A$ is an unknown amplitude and $\tau$ is an unknown delay.

The PSD of a linearly modulated signal scales as the magnitude squared of the spectrum of the modulating pulse. The time averages of the signal are the random process as a collection of deterministic signals, or realizations, evolving over time, where the specific realization is chosen randomly.

For a WSS process, the definitions of autocorrelation functions give the same result, which gives us the choice of computing the autocorrelation function and hence the PSD as either a time average or a statistical average. If all bits take the same value over a realization, then the waveform is simply a constant taking value +1 or -1 : clearly, a time average across such a degenerate realization does not yield typical results.

\section{Simulation Result}

In this section, we present the performance of the selection method of possible code words. Figures 1 to 8 depict the error performances of the $(64,42,8)$ RM code, the $(128,99,10)$ extended BCH code and the $(128,64,22)$ extended BCH code, respectively.

For each code, the symbols going through a transmit filter, a channel and a receive filter. Since symbols are being fed into the transmit filter, it is natural to expect that we can process the received signal such that, in the absence of channel distortions and noise, samples equal the transmitted symbols.

It is immediately obvious that the time domain condition gives the desired inter-symbol interference [5,6] avoidance. It can be shown that this is equivalent to the frequency domain condition by demonstrating that the sequence is the Fourier series for the periodic waveform obtained by summing all the aliased copies of the Fourier transform.

Thus, for the sequence to be a discrete delta, the periodic function must be a constant. The faster time decay compared with the pulse is evident from a comparison of the figures. The Nyquist criterion [7] applies to the cascade of the channel and receive filters. 


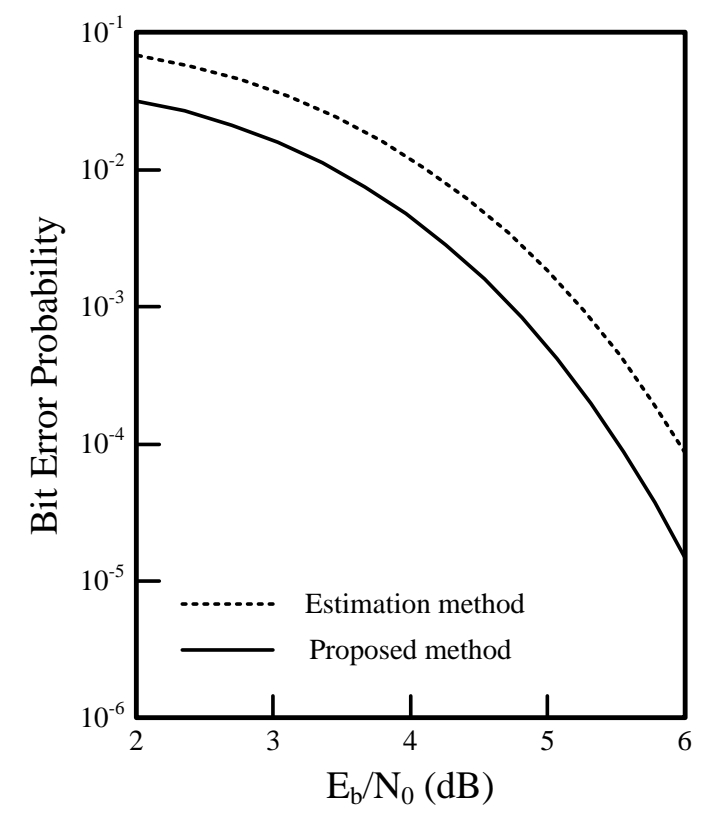

Figure 1. Bit error probability of the $(64,42,8)$ RM code with limits

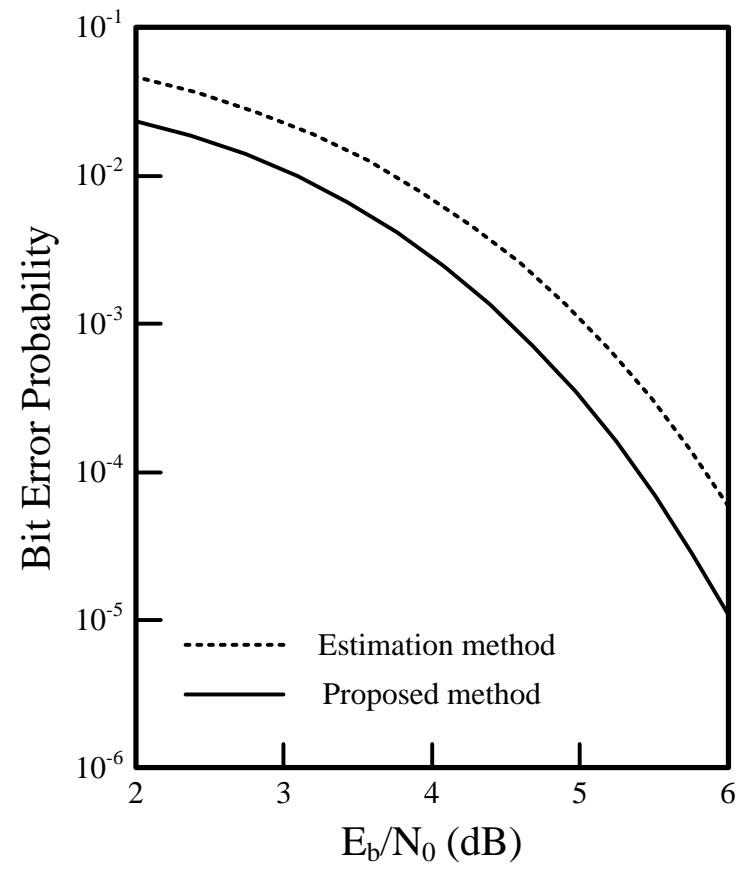

Figure 2. Bit error probability of the $(64,42,8)$ RM code without limits 


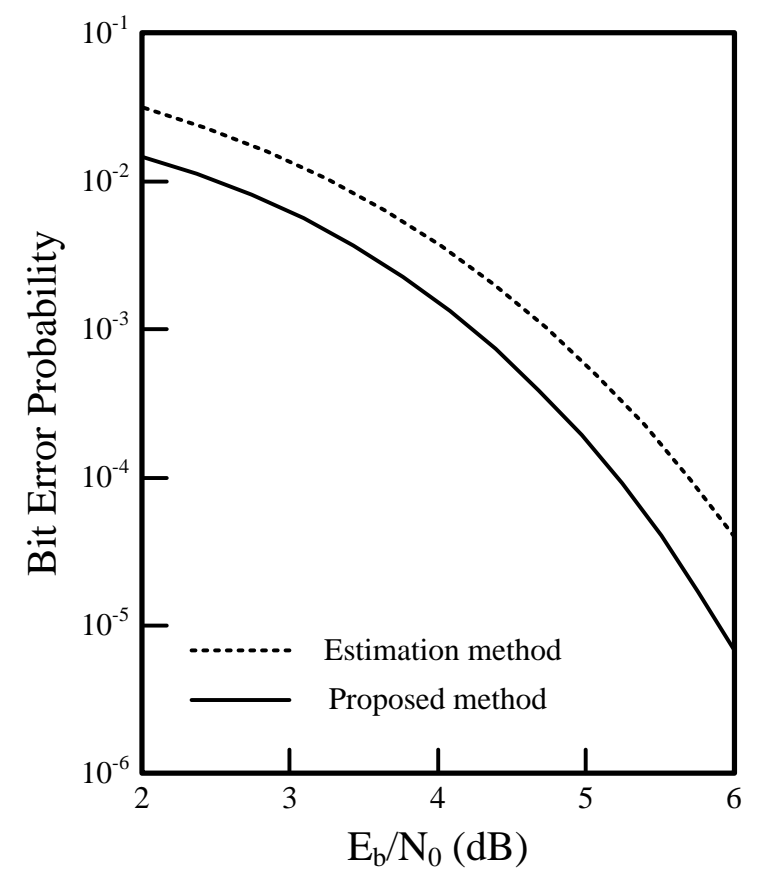

Figure 3. Bit error probability of $(128,99,10)$ extended BCH code

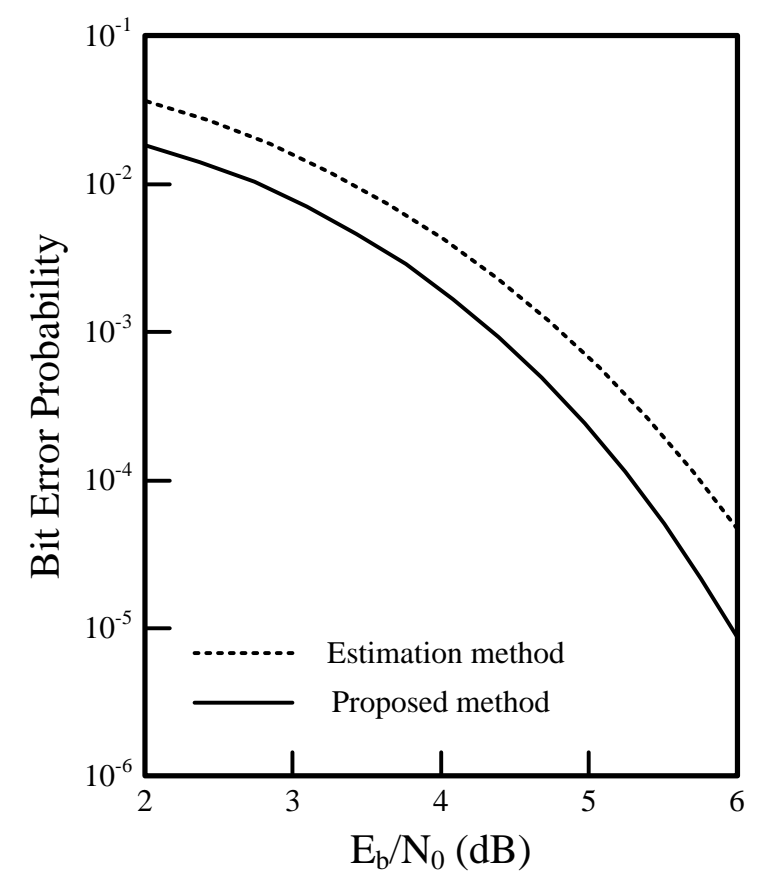

Figure 4. Bit error probability of $(128,64,22)$ extended $\mathrm{BCH}$ code 


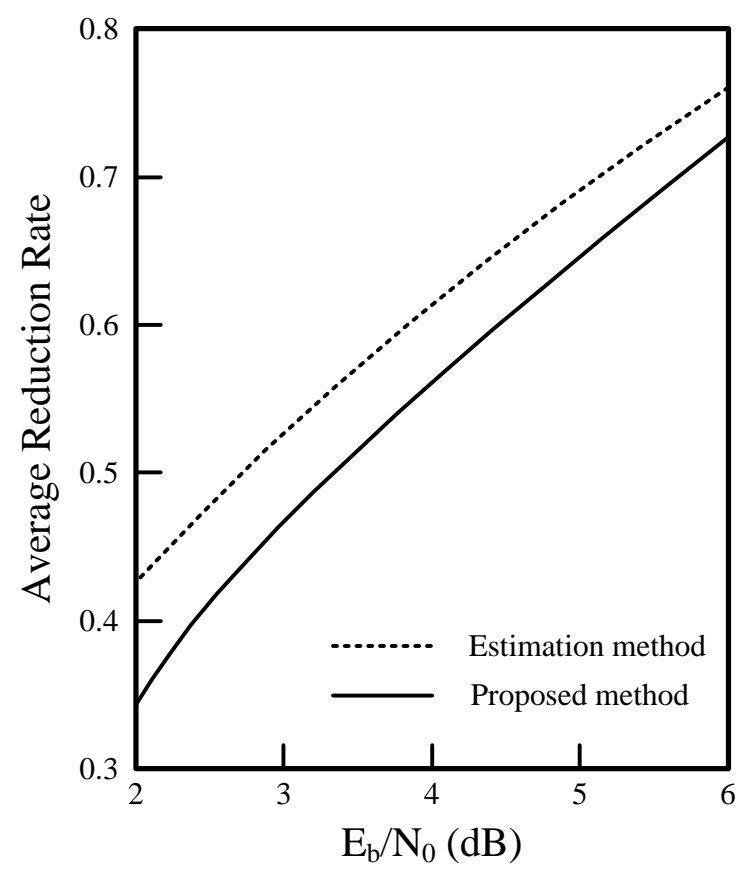

Figure 5. The average reduction rate of the $(64,42,8) \mathrm{RM}$ code

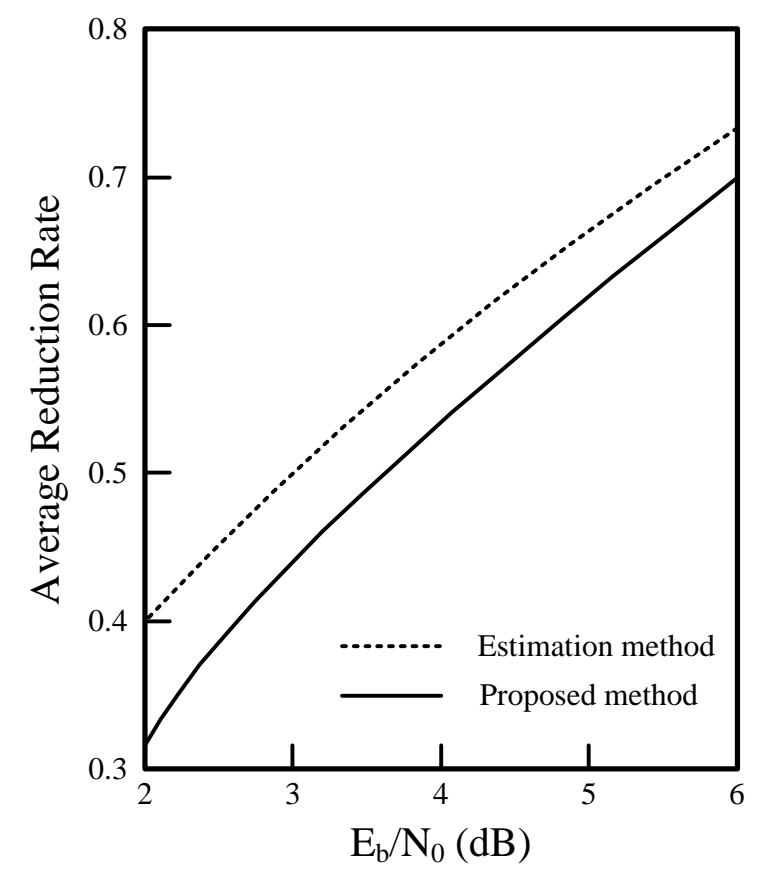

Figure 6 . The average reduction rate of $(128,99,10)$ extended $\mathrm{BCH}$ code 


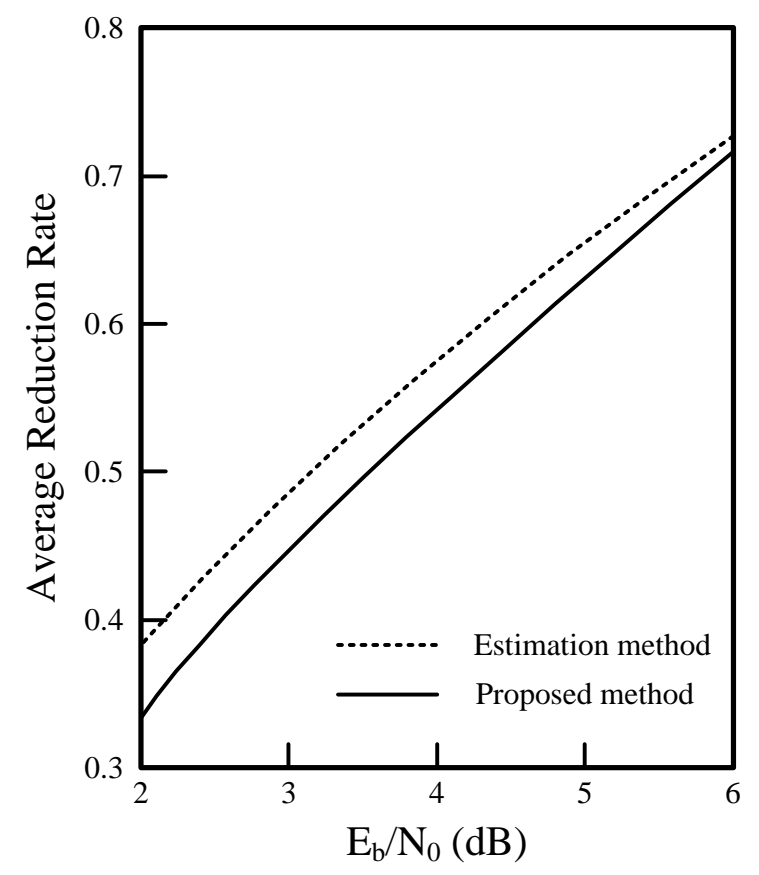

Figure 7. The average reduction rate of $(128,64,22)$ extended $\mathrm{BCH}$ code

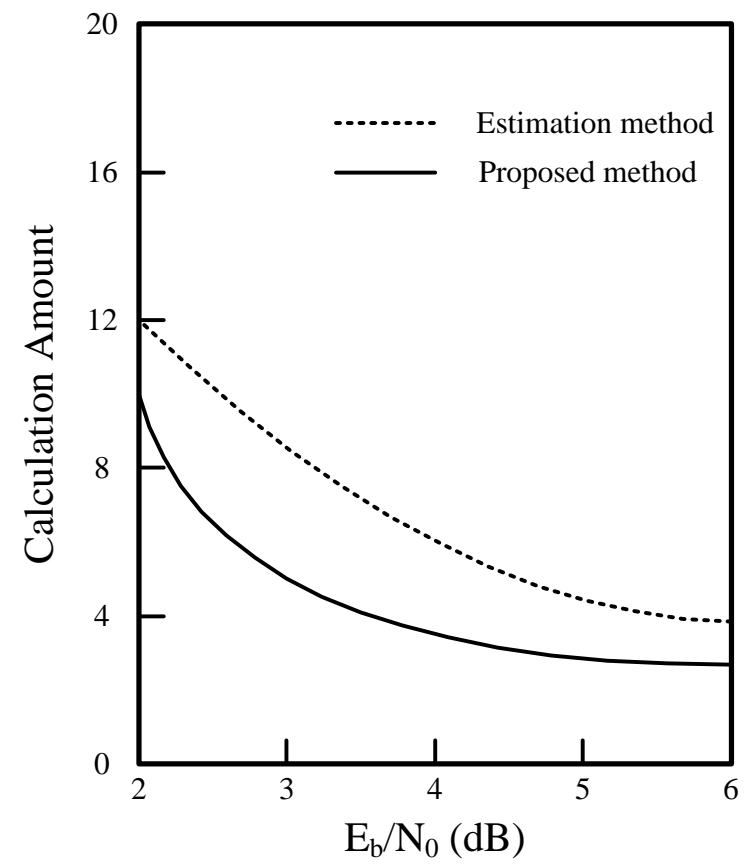

Figure 8. The calculation amount of the $(64,42,8) \mathrm{RM}$ code 


\section{Conclusion}

In this paper, a selection method of possible code words in channel coding systems is presented. The proposed method is for channel coding of digital communication systems. Measuring the error correction capability with reunion junction, the proposed method has superior performance and low implementation complexity.

Given the equivalence of pass-band and complex baseband, baseband operations can be efficiently performed in DSP. These digital algorithms are independent of the pass-band over which communication eventually occurs, and are amenable to a variety of low-cost implementations, including very large scale integrated circuits, field programmable gate arrays, and general purpose DSP engines.

\section{References}

[1] A. Balla, "Designing Pedagogical Learning Environment", International Journal of Advanced Science and Technology, vol. 6, (2009), pp. 1-14.

[2] A. Chennamaneni and J. T. C. Teng, "An Integrated Framework for Effective Tacit Knowledge Transfer", AMCIS, (2011).

[3] H. M. Campbell, "The liberation of Intellectual Capital through the natural evolution of Knowledge Management Systems", IEEE, (2010).

[4] H. Harlow, "The effect of tacit knowledge on firm Performance", Journal of Knowledge Management, vol. 12, no. $1,(\mathbf{2 0 0 8})$, pp. 148-163.

[5] C. A. Carver, R. A. Howard and E. Lavelle, "Enhancing student learning by incorporating learning styles into adaptive hypermedia", Proceedings of ED-MEDIA '96World Conf. on Educational Multimedia and Hypermedia, (1996) June, 17-22, Boston, USA.

[6] J. E. Gilbert and C. Y. Han, "Adapting instruction in search of a significant difference", Journal of Network and Computer Applications, vol. 22, (1999).

[7] P. Drucker, "The Age of Discontinuity: Guidelines to Our Changing Society", New York: Harper \& Row, (1968).

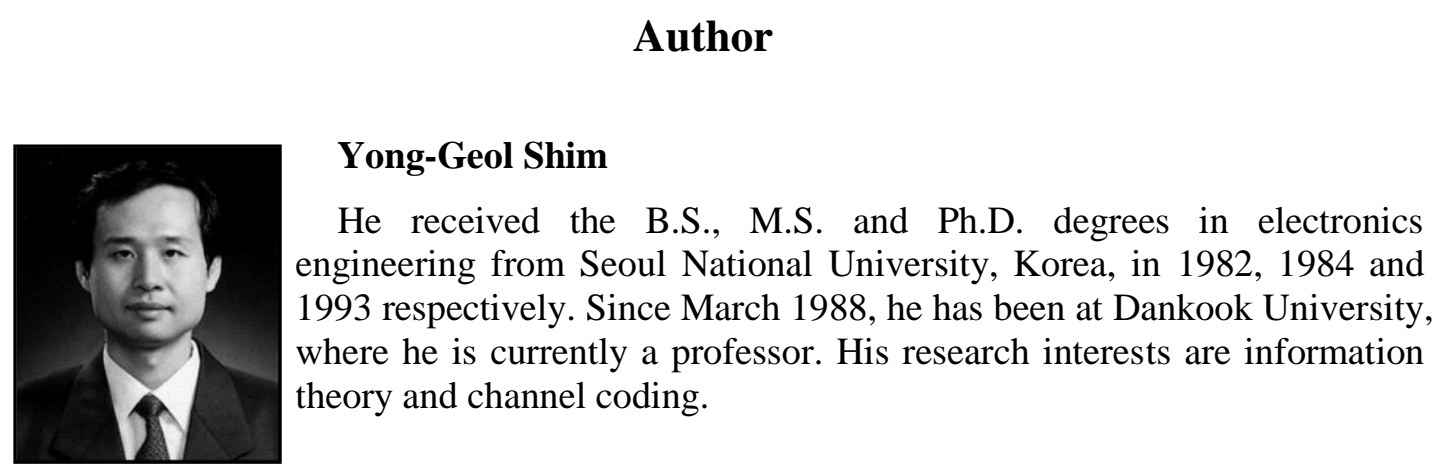

